\title{
FACTORES RELACIONADOS CON LA FALTA DE CONCIENCIA DE LOS DÉFICIT EN EL DAÑO CEREBRAL
}

\section{LACK OF AWARENESS IN TBI AND RELATED FACTORS}

\author{
Begoña González, Nuria Paúl, Juan luis BlázQuez, Marcos Ríos \\ Unidad de Daño Cerebral. Hospital Beata María Ana. C/Vaquerias, s/n. 28007. Madrid \\ e-mail: bekigonzalez@hotmail.com
}

\section{Resumen}

Se investigó la influencia de distintos factores en la manifestación de falta de conciencia de los déficit tras sufrir un daño cerebral. Los factores estudiados fueron localización hemisférica de la lesión (derecha e izquierda) y localización anatómica de la lesión, por un lado y la relación de la severidad inicial de la lesión y el tiempo transcurrido desde el daño cerebral, por otro. Ochenta y seis pacientes de la Unidad de Daño Cerebral del Hospital Beata M. ${ }^{a}$ Ana y sus familiares rellenaron el cuestionario "Índice de Competencia del Paciente». Los resultados mostraron que un sesenta y seis por ciento de los pacientes puntuaban como más eficaces sus competencias conductuales frente a la valoración de sus familiares. No parece existir una relación con la localización anatómica específica de la lesión, pero si con la severidad inicial de la lesión, manteniéndose dicha alteración transcurrido el primer año.

\section{Palabras Clave} déficit.

\begin{abstract}
Our purpose was to investigate the influence of several factors in the manifestation of impaired awareness following brain injury. We focused on the following factors, at one side the brain injury hemispheric side (right or left) and its neuroanatomical location, and on the other hand, the relation between initial severity and time post-injury. Participants were 86 patients with brain injury and their relatives recruited from the Brain Damage Unity, Beata M. ${ }^{a}$ Ana de Jesús Hospital. All of them were administered the questionnaire "patient competency rating scale». Assessment data showed that $66 \%$ of the patients perceived their behavioural abilities as more effective than their families' valuation. Lack of awareness did not correlate with injury location, but so with injury initial severity, sustaining after the following year.
\end{abstract}

\section{Key Words}

Brain injury, lack of awareness. 
Las consecuencias derivadas tras un daño cerebral adquirido (DCA) pueden ser múltiples y variadas dependiendo de diversos factores como la severidad de la lesión, las características del daño cerebral y de las zonas afectadas (tamaño y localización), características premórbidas del sujeto, etc. (Fernández y Perea Bartolomé, 2001). Tras la lesión se pueden presentar alteraciones tanto físicas, cognitivas, conductuales y emocionales. Entre las secuelas de tipo cognitivo, las más frecuentes incluyen dificultades de memoria, falta de concentración y fatiga, reducción de la velocidad para procesar la información, enlentecimiento motor, deterioro de la capacidad de planificación y solución de problemas y falta de conciencia de los déficit (FCD)(Muñoz Céspedes, 1999). Este último ha sido considerado uno de los factores predictivos de recuperación tras el daño cerebral (Jehkonen, Ahonen, Dastidar, Laippala y Vilkki, 2000; Jehkonen et al. 2001; Noé, Ferri, Simó y Chirivella, 2003; Hartman Maeir, Soroker, Oman y Katz, 2003).

Durante décadas se ha intentado establecer una definición clara sobre conciencia, sin embargo no se ha llegado al establecimiento de una definición común. La conceptualización más usada en la literatura, establecida por Prigatano y Schacter (1991), es considerar la conciencia como la capacidad para percibirnos a nosotros mismos de forma relativamente objetiva, manteniendo un sentido de subjetividad.

Para Stuss (1991), la conciencia ha sido definida como un atributo humano que no sólo permite tener conciencia de uno mismo, sino también darse cuenta de la posición del yo en el entorno social. Sin embargo a pesar de los múltiples intentos por establecer una definición de conciencia diversos autores mantienen la idea de que la conciencia no es un fenómeno unitario, sino que además implica varios niveles de complejidad (Tirapu, Muñoz y Pelegrín, 2003).

Finalmente una última definición de conciencia es la establecida por Ruiz -Vargas J. M (2000), donde la conciencia es considerada un estado de los organismos, determinado por las propiedades cerebrales y por el estado general de la persona en un momento específico, así mismo se pueden distinguir varios grados y formas de conciencia. Una manifestación parti- cular de este estado de conciencia sería la experiencia consciente, referida al "ser consciente de algo», es decir, "Tener una experiencia subjetiva concreta que está determinada por el estado de conciencia actual y la estimulación actual externa e interna» (pp. 355).

Aplicado al daño cerebral, la falta de conciencia de las limitaciones pueden ser inconsistentes entre los dominios físicos, cognitivos y afectivos (Visser, Meyboom, Deelman, Berg y Gerritsen, 2002), ya que una misma persona puede reconocer sus déficit o secuelas físicas, pero no las de tipo cognitivo o emocional, o viceversa. Algunos estudios encuentran una mayor falta de conciencia de los déficit de tipo físico frente a los de tipo emocional o comportamental (Prigatano, Altaman y O’Brien, 1991; Hartman Maeir, Soroker, Ring y Katz, 2002).

Para otros autores como Chittum, (1996), la autoconciencia varia a lo largo de un continuo, desde reconocer que una determinada capacidad ha disminuido hasta ser capaz de anticipar dificultades futuras debido a los déficit de la persona y buscar soluciones alternativas.

En la misma línea, Fleming y Strong (1995) propusieron un modelo de definición de la conciencia en relación con el daño cerebral, usando para ello una clasificación en tres niveles diferentes de falta de conciencia, el primero de ellos hace referencia al conocimiento objetivo o conciencia de los déficit, es decir, implicaría que la persona conozca que una determinada capacidad ha disminuido. El segundo nivel de conciencia supone el conocimiento de las implicaciones funcionales que los déficit tienen sobre el funcionamiento en la vida diaria, en este nivel no es suficiente con saber que una capacidad ha disminuido, sino también conocer que repercusiones ha tenido en el funcionamiento cotidiano de la persona. Y el tercer nivel hace mención a la habilidad para el establecimiento de objetivos realistas a corto y largo plazo teniendo en consideración los déficit presentes y utilizando estrategias alternativas de adaptación.

Los cuestionarios diseñados para la valoración de conciencia de los déficit, evalúan únicamente el primer nivel de conciencia, también llamada, «Conciencia Intelectual», para estudiar 
el resto de niveles de conciencia será necesario observar el comportamiento del paciente en diferentes situaciones (García, Sánchez, Urrutikoetxea, Muñoz Céspedes y Quemada, 2003).

Aplicado al daño cerebral, la FCD es una de las alteraciones neuroconductuales frecuentemente observadas en pacientes que han sufrido daño cerebral traumático, accidente cerebrovascular u otro tipo de patologías con afectación sobre el Sistema Nervioso Central (Wagner, Spangenberg, Bachman y O'Connell, 1997; Anderson y Tranel, 1989). La prevalencia de este déficit tras sufrir un daño cerebral oscila, según diversos estudios, entre un 33\% y un 52\%, (Starkstein, Fedoroff, Price, Leiguarda, y Robinson, 1993; Hartman Maeir, Soroker et al. 2003) y como señalan Oddy, Coughlan, Tyerman y Jenkins (1985) en un 52\% de los afectados, se mantenía incluso siete años después de la lesión. Así mismo otros autores mantienen que la FCD tras daño cerebral desaparece a los tres meses tras la lesión y su mantenimiento tras este periodo representa un importante factor predictivo de pobre recuperación (Jehkonen, Ahonen et al. 2000). Del mismo modo otro de los factores relacionados con la presencia y mantenimiento de la FCD a largo plazo ha sido la severidad inicial de la lesión, considerada como la puntuación en la Escala de Coma de Glasgow (GCS) (Teasdale y Jennett, 1974). Dicha escala fue diseñada para valorar la severidad inicial de la lesión, predecir la recuperación del paciente y observar la evolución del estado de coma. Para ello, se atiende a tres parámetros: Apertura de ojos, Respuesta motora y Respuesta verbal.

La puntuación de la escala oscila entre tres y quince puntos, dónde una puntuación menor o igual a ocho indica daño cerebral severo, entre nueve y doce puntos daño cerebral moderado y entre trece y quince puntos daño cerebral leve. En un estudio realizado por Prigatano, Bruna, Mataro, Muñoz Céspedes, Fernández y Junque (1998), con una muestra de población española de pacientes que habían sufrido TCE y utilizando el cuestionario descrito en esta investigación: Índice de Competencia del Paciente, observan que un $40 \%$ de los pacientes afectados por daño cerebral severo, determinado a partir de la GCS, tendían a sobrestimar sus competencias con- ductuales, característica relacionada con lo que se ha considerado como FCD. En dicho estudio se concluye que la severidad inicial de la lesión, parece relacionarse con la posterior presencia de FCD.

Las FCD puede tener importantes implicaciones sobre el funcionamiento de la persona en su vida cotidiana, siendo lo suficientemente relevantes como para prestar una mención especial durante el proceso de valoración. A nivel conductual, los pacientes pueden manifestar despreocupación, pobre autocontrol, autorregulación deficiente de la conducta y fracaso en la interacción con el entorno (García, Sánchez et al. 2003). Por lo que respecta a la recuperación tras la lesión, la FCD juega un papel primordial a la hora de obtener beneficios durante el periodo de rehabilitación, ya que su presencia constituye un importante obstáculo para el proceso de rehabilitación (Hartman Maeir, Soroker et al. 2002), manifestado por una baja motivación y resistencia al tratamiento (Fleming, Strong y Ashton, 1996), pobre participación en las actividades terapéuticas y expectativas inadecuadas en cuanto a sus capacidades y las actividades que pueden realizar (Toglia y Kirk, 2000).

Noé, Ferri et al. (2003), en un estudio con población española en el que valoraron la implicación de la FCD sobre la eficacia del programa de rehabilitación, en personas que habían sufrido DCA, concluyeron que la adecuada conciencia de los déficit resultantes tras un DCA es un indicador clínico fiable y un válido marcador pronóstico de la situación cognitiva, psicopatológica y funcional del paciente con DCA.

Así mismo tras el proceso de rehabilitación la FCD constituye un impedimento para la reincorporación a la vida social, laboral y familiar de la persona.

En cuanto a la readaptación laboral, la FCD conlleva una serie de riesgos relacionados con la efectividad del trabajo realizado y el mantenimiento del empleo, entre ellas, se pueden citar la falta de uso de las medidas de seguridad, aislamiento social, escasa implicación y responsabilidad, falta de autonomía en el trabajo, irritabilidad o expectativas poco realistas (Muñoz Céspedes, Ríos, Ruano y Moreno, 1998; Muñoz 
Céspedes, Ríos, Ruano y Moreno, 1999; Muñoz Céspedes, Paúl, Pelegrín y Tirapu, 2001).

Dada la importante repercusión que la FCD puede tener sobre el proceso de rehabilitación y sobre la calidad de vida de la persona afectada por DCA, diversos autores han postulado diversos modelos teóricos y descrito factores explicativos que pudieran relacionarse con la presencia de esta alteración. Entre ellos se pueden distinguir aquellos que relacionan FCD con lesiones predominantemente hemisféricas derechas, otros estudios donde las regiones anatómicas responsables son las áreas frontales, otros autores defienden la idea de la presencia de alteraciones mnésicas asociadas a tal manifestación y finalmente otros autores defienden la ausencia de relación con una localización anatómica específica. Brevemente describo las investigaciones más relevantes que respaldan estas teorías.

Langer y Padrone (1992), establecieron una clasificación basándose en las diferentes causas que pueden dar lugar a una manifestación de FCD. Por un lado la falta de conciencia puede ser consecuencia directa del daño cerebral, caracterizada por alteraciones neuropsicológicas y nulas implicaciones emocionales, el segundo nivel implicaría una minimización de los déficit, explicados en parte por déficit neuropsicológicos y por mecanismos psicológicos, finalmente el tercer nivel se caracteriza por una negación de los déficit presentes en ausencia de alteraciones neuropsicológicas que pudieran explicarlo. En la misma línea Giacino y Cicerone, (1998), definieron una variedad de déficit de falta de conciencia, algunos como consecuencia directa del daño cerebral, frente a otros determinados a partir de mecanismos psicológicos de negación de los déficit, ya que en ocasiones la depresión puede inducir a la persona a sobreestimar sus competencias conductuales (Prigatano y Altman, 1990). Esto sugiere la necesidad de establecer una distinción entre la presencia de esta alteración de falta de conciencia como causa neurológica, frente a otro tipo de alteración cuya causa es de tipo psiquiátrico. Esta diferenciación parece ser relevante de cara al establecimiento de una programa adecuado de rehabilitación, diferenciado en cada caso particular.

Visser, Meyboom de Jong et al, (2002) analizaron los cambios en aspectos emocionales, cognitivos y conductuales juzgados por el paciente y su familiar, en sujetos tras sufrir un accidente cerebrovascular. Estos autores observaron que los sujetos con lesiones en hemisferio derecho consideraban como menos frecuentes y severos los cambios sufridos frente a la opinión de sus familiares. Este grupo fue comparado con sujetos cuyas lesiones fueron localizadas en el hemisferio izquierdo y donde el acuerdo, respecto a los cambios sufridos tras el accidente, con sus familiares fue mayor. Esta hipótesis del hemisferio derecho esta basada en una gran cantidad de estudios que sugieren que las lesiones derechas son comunes en casos donde se manifiesta anosognosia para hemiplejia u otro tipo de alteraciones de conciencia (Prigatano y Altman, 1990).

Por otro lado entre los autores que defienden la idea de la presencia de daño en regiones frontales asociados a FCD. Stuss (1991) hace referencia a los primeros casos descritos en la literatura donde pacientes con lesiones frontales parecían comprender la seriedad de su situación tras las lesiones sufridas y sin embargo no mostraban un interés real hacia la gravedad de su situación. Esta descripción parece ser congruente con lo que hoy se ha descrito como una falta de conciencia de las consecuencias e implicaciones futuras que las lesiones sufridas tienen sobre el funcionamiento de la persona. Descripciones similares fueron hechas por varios autores a lo largo de los años (Sachs, 1927, German y Fox's 1932, Brickner's 1936). Todos ellos hacen especial hincapié en destacar la indiferencia afectiva o falta de preocupación hacia su enfermedad y las consecuencias que conlleva, como la característica más destacable tras la lesión cerebral en regiones frontales.

Del mismo modo Luria y Homskaya, (1964) y Luria, (1969) tras la observación de diversos casos de falta de conciencia de los déficit y lesiones frontales establecieron dos síntomas presentes en la mayoría de los pacientes tras sufrir estas lesiones: manifestaban una alteración sobre el comportamiento intencional y una deficiente actitud critica hacia su propio comportamiento.

Por otro lado algunos autores sugieren que la FCD va asociado a la presencia de desorientación y déficit de memoria, implicando un re- 
quisito imprescindible para la presencia de falta de conciencia (Johnson, 1991); sin embargo otros estudios no parecen apoyar esta idea, como es el caso de la investigación realizada por Starkstein, Fedoroff et al. (1993), en el que se comparó a pacientes con FCD y sin ella, en relación con la presencia de déficit neuropsicológicos y donde se concluye que la disfunción frontal, pero no la de memoria, podría jugar un papel importante en la producción de FCD, datos, así mismo, apoyados por otras investigaciones (Prigatano y Altman, 1990).

Giacono y Cicerone (1998) mencionan tres posibles factores explicativos para la aparición de falta de conciencia de los déficit tras daño cerebral. El primero de ellos lo relacionan con la presencia de un déficit de memoria, ya que para ser conscientes de los déficit, el paciente debe realizar una comparación entre su funcionamiento anterior y el actual. Sin embargo, como se ha comentado anteriormente diversas investigaciones contradicen la implicación del deterioro mnésico en la FCD. El segundo de los factores, sugiere que la FCD es un mecanismo psicológico de defensa por el que las consecuencias del daño cerebral son negadas por el paciente. Sin embargo, los autores no explican porque existe una mayor proporción de FCD tras lesiones derecha, y por qué algunos déficit son negados y otros no (Hartman Maeir, Soroker, Oman y Katz, 2001).

La tercera hipótesis explica la FCD como consecuencia directa del daño cerebral, con una mayor incidencia de lesiones derechas frente a izquierdas (Visser, Meyboom de Jong et al, 2002).

Finalmente, McGlynn y Schacter (1989), basándose en el modelo teórico de Mesulam (1985), propusieron diferentes formas de falta de conciencia, así las lesiones bilaterales del lóbulo frontal darían lugar a FCD del comportamiento social y funciones ejecutivas, las lesiones parietales inferiores, estarían relacionadas con una FCD de la percepción espacial y finalmente las lesiones temporales izquierdas, con una reducida capacidad para percibir dificultades del lenguaje. Todas estas áreas relacionadas con FCD, son las denominadas por Mesulam como córtex heteromodal (Mesulam, 1985). Estas regiones son conocidas por su capacidad para res- ponder al input de múltiples estímulos, así mismo tienen conexiones recíprocas con diversas estructuras cerebrales, importantes en la integración de información cognitiva y emocional En la misma línea Prigatano y Altman, (1990) afirman que el deterioro bilateral del córtex heteromodal puede jugar un papel relevante tras varios años después de la lesión, haciendo hincapié en la ausencia de relación entre una localización anatómica específica y la posterior presencia de FCD (Prigatano y Altman, 1990; Prigatano, 1996; Hartman Maeir, Soroker et al. 2001). Estos datos parecen sugerir que las lesiones bilaterales tienen una peor recuperación sobre la FCD, datos que podrían ser relevantes de cara al establecimiento de un pronostico y a la hora de establecer los objetivos de los programas de rehabilitación.

En este estudio se pretende ampliar el conocimiento de los factores implicados en la aparición de falta de conciencia de déficit tras un daño cerebral adquirido. Los objetivos planteados son cuatro, entre ellos se encuentran analizar la frecuencia de FCD en una muestra de pacientes con daño cerebral, observar el papel que juega la severidad inicial de la lesión en la posterior manifestación de FCD, así como el tiempo transcurrido desde la lesión con la recuperación progresiva de una conciencia adecuada de las limitaciones y finalmente analizar una posible relación de presencia de FCD con la localización hemisférica de la lesión.

A partir de dichos objetivos se han establecido las siguientes hipótesis:

1. Al menos, un 33\% de los afectados por DCA mostrarán diferencias en más de cinco puntos en el cuestionario al ser comparado con sus familiares (Starkstein, Fedoroff et al. 1993; Prigatano et al. 1998; Hartman Maeir, Soroker et al. 2003).

2. Los pacientes con lesiones localizadas en el hemisferio derecho mostrarán mayores diferencias en el cuestionario PCRS al ser comparado con su familiar, frente a los pacientes afectados por lesiones izquierdas. (Anderson y Tranel, 1989; Visser, Meyboom de Jong et al. 2002).

3. La severidad de la lesión, definida a partir de la valoración inicial en GCS, está rela- 
cionada con la presencia de FCD, entendido como una menor puntuación en la GCS se asociará con mayores diferencias en el cuestionario entre el paciente y su familiar (Giacino y Cicerone, 1998; Prigatano et al. 1998; Hartman Maeir, Soroker, et al. 2003).

4. La conciencia de las limitaciones se irá adquiriendo de forma progresiva, existiendo una relación negativa entre el tiempo transcurrido desde la lesión y la FCD, es decir, a mayor tiempo transcurrido desde la lesión menor presencia de FCD en la muestra objeto de estudio, sin embargo, se mantendrá un año después del accidente en, al menos, un $52 \%$ de los sujetos con inicial FCD (Oddy, Coughlan et al. 1985; Fleming, Strong y Ashton, 1998).

\section{Método}

\section{Participantes}

Ochenta y seis pacientes afectados por daño cerebral adquirido intervinieron en el estudio, de los cuales $63(73,3 \%)$ fueron hombres y 23 $(26,7 \%)$ mujeres. Todos los pacientes acudían a la Unidad de Daño Cerebral del Hospital Beata M. ${ }^{a}$ Ana de Madrid para seguir tratamiento rehabilitador, como consecuencia de diversas patologías (Traumatismo Craneoencefálico (TCE), Accidente Cerebrovascular (ACV) y Encefalitis).

Se calcularon las frecuencia, medias y desviaciones típicas de las variables demográficas de la muestra (edad, tiempo trascurrido desde la lesión, severidad inicial y etiología de la lesión). Los datos se recogen en la tabla 1 y figura 1.

TABLA 1. Datos demográficos de la muestra

\begin{tabular}{|l|c|c|c|c|}
\hline & Mínimo & Máximo & Media & Desv. típ. \\
\hline Edad & 14 & 76 & 37,45 & 15,619 \\
\hline Tiempo desde la lesión (meses) & 2 & 58 & 13,32 & 10,983 \\
\hline Escala de Coma de Glasgow & 3 & 15 & 5,8 & 2,68 \\
\hline N & 86 & & & \\
\hline
\end{tabular}

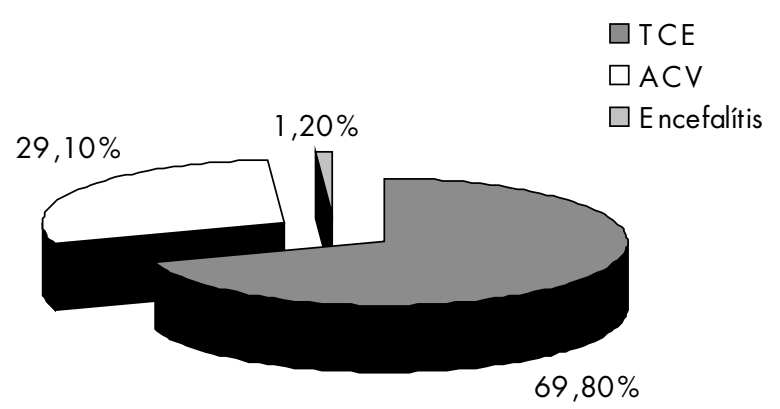

Figura 1. Etiología de la lesión

La severidad de la lesión se determinó a partir de la puntuación inicial en la Escala de Coma de Glasgow (GCS) (Teasdale y Jennett, 1974). Según esta escala, $58(87,9 \%)$ pacientes sufrieron un daño cerebral severo (puntuación menor o igual a ocho en la GCS), seis $(9,1 \%)$ daño cerebral moderado (puntuación entre nueve y 12) y dos $(3 \%)$ daño cerebral leve (puntuación entre 13 y 15).

En relación con el nivel educativo, 25 pacientes $(29,8 \%)$ tenían estudios primarios, 19 $(22,6 \%)$ secundarios, 16 (19\%) habían cursado estudios universitarios de grado medio y 24 $(28,6 \%)$ de grado superior. En la mayoría de los casos el familiar que rellenó el cuestionario, en la modalidad familiar, fue el cónyuge $(38,8$ $\%)$ o la madre $(31,8 \%) /$ padre $(11,8 \%)$ del afectado.

No se encontraron diferencias significativas en función del sexo $(\mathrm{t}=1.355, \mathrm{p}<.05)$, nivel educativo $(\mathrm{F}=.688, \mathrm{p}<.05)$, edad $(\mathrm{F}=1.216, \mathrm{p}<.05) \mathrm{y}$ etiología $(\mathrm{t}=.183, \mathrm{p}<.05)$ en las puntuaciones entre el paciente y su familiar en el cuestionario. 
A partir de la Historia clínica y de los resultados obtenidos por técnicas de neuroimagen traídos por el paciente a la unidad, se recogieron los datos relacionados con la localización anatómica de la lesión, descritos en las figuras 2 y 3.

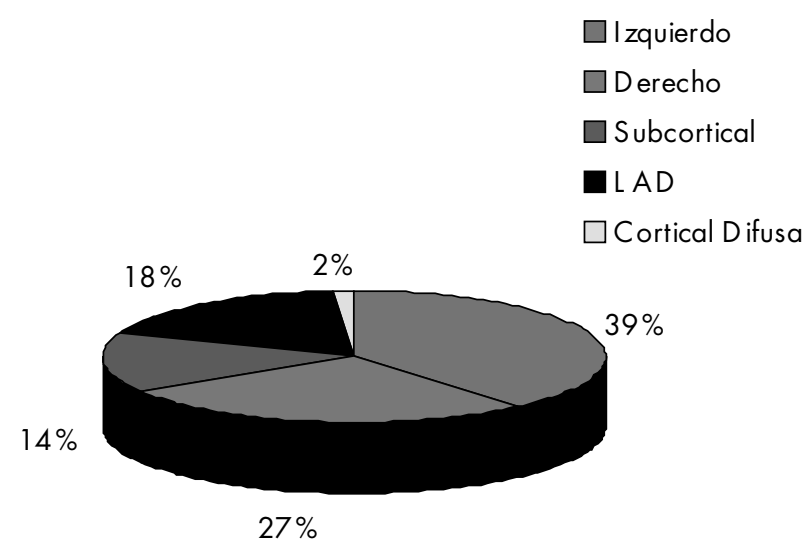

FIGURA 2. localización de la lesión

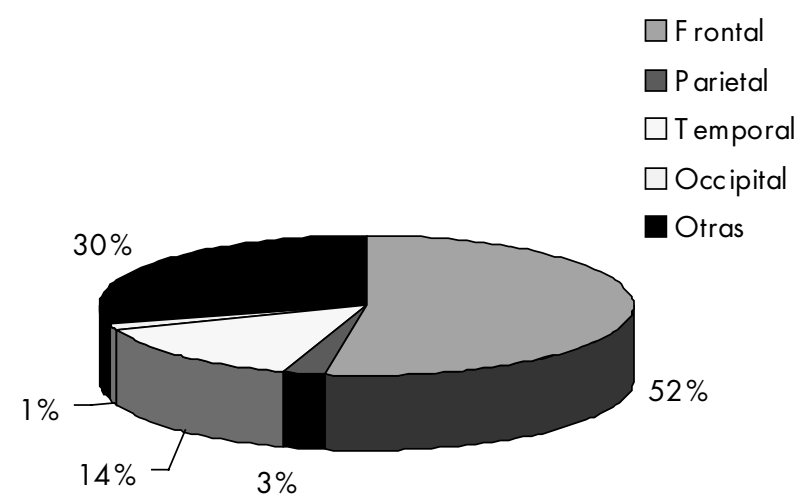

FiguRa 3. Localización de la lesión por lóbulos

Todos los pacientes incluidos en la muestra presentaban un nivel adecuado de habilidades lingüísticas para completar el cuestionario. Se excluyeron aquellos sujetos que presentaban alteraciones importantes a nivel lingüístico, que impidiese la correcta interpretación y respuesta de los ítem del cuestionario.

\section{Material}

Para la valoración de la conciencia de las limitaciones tras daño cerebral se utilizó la traducción española: Índice de Competencia del Paciente (ICP) (Prigatano et al. 1998) del cues- tionario Patient Competency Rating Scale (PCRS) (Prigatano et al, 1986). El ICP es el principal cuestionario de valoración de la FCD que ha sido aplicado a población española, estudio realizado por Prigatano et al. (1998) en el que, utilizando el ICP con población afectada por DCA, concluyeron que un $40 \%$ de los afectado tendían a sobrestimar sus competencias conductuales.

El ICP es una escala formada por 30 ítem referidos a diferentes actividades de la vida cotidiana, entre las que se incluyen actividades básicas e instrumentales de la vida diaria, habilidades sociales, aspectos cognitivos y emocionales. Para responder se utiliza una escala likert de cinco puntos en función del nivel de dificultad que la persona juzga para realizar estas actividades, donde una puntuación de uno indica «No puedo hacerlo», hasta cinco interpretado como «Puedo hacerlo con facilidad «. Tiene dos versiones para ser administrada al paciente y a un observador (generalmente un familiar). La identificación de FCD viene determinada por la diferencia en las valoraciones hechas por el paciente frente al familiar. El uso de este tipo de cuestionarios en los que se contrasta la opinión del paciente con la de otra fuente considerada más objetiva, ha sido la forma de valoración más común para identificar la presencia de FCD tras daño cerebral, específicamente el uso del PCRS frente a otro tipo de cuestionarios similares. (Deaton, 1986; Prigatano y Altman, 1990; Prigatano, Altman et al. 1991; Fleming y Strong, 1995; Fleming, Strong et al. 1996; Prigatano et al. 1998; García, Urrutikoetxea, Muñoz Céspedes y Quemada, 1999; Sherer, Hart y Nick, 2003; García, Sánchez et al. 2003)

Para determinar la presencia o ausencia de conciencia de déficit, a partir de las diferencias en el cuestionario en paciente y familiar, se contrastó el promedio de puntuaciones obtenidas por los pacientes y sus familiares. Así mismo se tomó como referencia el punto de corte utilizado en varios estudios (Prigatano et al., 1998; Hartman Maeir, Soroker et al., 2003), a partir de los cuales se considera que el paciente sobreestima o presenta falta de conciencia de sus limitaciones cuando las diferencia de puntuaciones en ambos cuestionarios son mayores a cinco puntos, con signo positivo, por otro lado la 
conciencia de las limitaciones es adecuada cuando la diferencia de puntuaciones se sitúan dentro del rango más/ menos cinco puntos, y finalmente una diferencia de puntuaciones mayores a cinco puntos con signo negativo, indican una subestimación de las capacidades por parte del paciente.

En cuanto a las propiedades psicométricas del PCRS, tanto en la modalidad de pacientes como en la de familiares, se obtuvo un alfa de Cronbach de 0.91-0.95, y un acuerdo pre- post test de 0.85- 0.97, respectivamente, datos que reflejan una buena consistencia interna y adecuada fiabilidad test- retest. (Prigatano, Altaman et al. 1991; Heilbronner, Millsaps, Azrin, Mittenberg, 1993).

En un estudio realizado por Sherer, Hart et al. (2003), se comparó el cuestionario PCRS y el Awareness Questionaire (AQ), cuestionario de frecuente uso para ser utilizado en la valoración de los déficit de conciencia tras daño cerebral. Los resultados mostraron correlaciones moderadas $\left(r_{s}: 0.50, p<0.01\right)$, entre ambos cuestionarios, concluyendo que tanto el PCR como el AQ, representan medidas comparables de FCD tras daño cerebral.

Así mismo también se observa una alta consistencia interna de la versión española del PCRS, con un alfa de Cronbach de 0.87 y 0.91 en el cuestionario de pacientes y familiares respectivamente (Muñoz Céspedes, Calvo, Ríos, García, Sánchez, 2002).

La escala ICP, en la modalidad del paciente y familiar, puede ser consultada en el Apéndice A.

\section{Procedimiento}

Se utilizó un diseño ex post facto restrospectivo. Se tuvieron en cuenta tres variables independientes: localización hemisférica de la lesión (derecha, izquierda), localización anatómica de la lesión (Frontal frente al resto de áreas: Temporal, Parietal y Occipital) y severidad de la lesión (Leve, Moderado y Severo). Como variable dependiente se midió las diferencias de puntuaciones entre el pacientes y su familiares (puntuación total del paciente menos la del familiar en el cuestionario ICP)
Se midieron y analizaron las variables demográficas para asegurarse que las diferencias encontradas en la presencia o ausencia de falta de conciencia de los déficit pudiera deberse a ellas.

La administración del cuestionario a los pacientes fue realizada de forma individual en la sala habitual del terapeuta y supervisada, en caso de duda en alguno de los ítem, por el profesionales habitual que trata a cada uno de los pacientes (Terapeutas ocupacionales y Neuropsicólogos).

Así mismo a los familiares se les proporcionó el cuestionario, en el formato para familiares, para ser rellenado siguiendo las instrucciones descritas en él.

\section{Resultados}

Se realizó una prueba t para muestras relacionadas, para observar las diferencias entre el cuestionario rellenado por el paciente y por el familiar. Se encontraron diferencias significativas entre la valoración de los pacientes y la de sus familiares en el cuestionario $(t=6.553, p<0.05)$. Para observar la frecuencia de aparición de falta de conciencia en la muestra, se calculó la diferencia entre las puntuaciones recogidas en el ICP completado por el paciente y el ICP completado por el familiar. A partir de la tabla de frecuencias y utilizando un punto de corte de mas/ menos cinco puntos, se observa que $57(66,3 \%)$ pacientes obtienen diferencias mayores a cinco puntos en el cuestionario, al ser comparado con el completado por su familiar, por otro lado 19 $(22,1 \%)$ pacientes mostraron diferencias menores a cinco puntos, y finalmente 10 pacientes $(11,6 \%)$ obtenían puntuaciones inferiores a sus familiares, dando lugar a una diferencia entre paciente y familiar de signo negativo.

Para analizar el papel que juega tanto la severidad de la lesión inicial como el tiempo transcurrido desde la lesión en la presencia de FCD, se calculó el coeficiente de correlación de Pearson. Se observa la presencia de una correlación estadísticamente significativa tanto entre la puntuación inicial en la GCS y la diferencia en el cuestionario entre paciente- familiar $(r=-0.273$; $\mathrm{p}=0.05$ ), como entre el tiempo transcurrido des- 
de la lesión y la diferencia en el cuestionario $(\mathrm{r}=$ - 0.310; $p=0.01$ ), ambas de signo negativo. Así mismo se encontraron diferencias significativas en función del tiempo transcurrido tras la lesión $(\mathrm{t}=2.443, \mathrm{p}<.05)$. Para observar la presencia de FCD a lo largo del tiempo tras la lesión, se realizó un análisis de las frecuencias. Del total de sujetos cuyas valoraciones se realizaron durante el primer año tras sufrir la lesión $(\mathrm{N}=51), 37$ $(72.5 \%)$ mostraban FCD y de los valorados transcurrido el primer año $(\mathrm{N}=33), 19(57.6 \%)$ continuaban mostrando FCD.

Finalmente se realizaron dos pruebas t para muestras independientes, para observar la posible implicación hemisférica (lesiones izquierdas frente a lesiones derechas)por un lado, y de las regiones frontales por otro (localización frontal frente al resto de localizaciones anatómicas: temporal, parietal, occipital) en la presencia de FCD, no encontrándose diferencias significativas entre lesiones izquierdas y derechas $(\mathrm{t}=0,437, \mathrm{p}<0,05)$ así mismo tampoco resultó significativa las diferencias $(\mathrm{t}=0.434$, $\mathrm{p}<0.05)$ al comparar los pacientes con lesiones frontales frente a las lesiones localizadas en otras áreas cerebrales (Temporal, Parietal y Occipital).

\section{Discusión}

En este estudio se investigó la frecuencia de aparición de FCD y el papel que juegan diferentes factores en la posterior manifestación de FCD tras sufrir un daño cerebral adquirido. Entre los factores analizados se encuentran la localización hemisférica de la lesión, derecha e izquierda, la implicación de lesiones en regiones frontales frente al resto de áreas lesionadas, la severidad inicial de la lesión, valorado a partir de la puntuación inicial en la escala de coma de Glasgow, y el tiempo transcurrido después de la lesión. Los datos obtenidos muestran que un $66 \%$ de la muestra de pacientes con daño cerebral adquirido estudiada, valoraban sus competencias conductuales de forma más eficaz frente a lo observado por su familiar. Así mismo no parece existir una localización específica de la lesión relacionada con la posterior presencia de FCD, sino que tal manifestación parece responder a la influencia de una serie de factores, entre ellos se puede citar la severidad inicial de la lesión y el tiempo transcurrido desde la lesión, los cuales correlacionan con la presencia de falta de conciencia de las limitaciones, de forma significativa y con signo negativo, esto es, a menor puntuación inicial en la GCS, mayor FCD posteriormente; del mismo modo el transcurso del tiempo tras la lesión está relacionado con menor presencia de FCD, sin embargo está alteración se mantiene en un 57.6\% de los sujetos valorados un año después de sufrir la lesión cerebral. Lo que parece indicar que el periodo de recuperación de la ausencia de conciencia de los déficit, requiere un periodo temporal mayor de un año en la mayoría de los casos.

En relación con la primera hipótesis, donde se planteaba que, al menos, un 33\% de los pacientes mostrarán FCD, los resultados son congruentes con los obtenidos por otros investigadores (Starkstein, Fedoroff et al. 1993; Hartman Maeir, Soroker et al. 2003), ya que del total de la muestra de estudio un 66\% presentan FCD.

Del mismo modo los datos relacionados con la influencia de variables anatómicas, contradicen las hipótesis propuestas inicialmente, en las que se planteaba una implicación hemisférica derecha. Así mismo tampoco se encontró una relación de áreas frontales en la posterior presencia de FCD. Estos datos parecen ser acordes con los encontrados por otros autores, donde se indica que la FCD no puede ser explicada en base a la localización de la lesión, sugiriendo una relación del deterioro bilateral y cortex heteromodal con la presencia de FCD (McGlynn y Schacter, 1989; Prigatano y Altman, 1990; Prigatano, 1996; Hartman Maeir, Soroker et al. 2001).

Finalmente, la severidad inicial de la lesión y el tiempo transcurrido, si parecen ser dos factores influyentes, aunque no determinantes, en la FCD, al encontrarse correlaciones, a pesar de ser bajas, significativas entre estas variables y la presencia de FCD. Esto parece indicar que la severidad de la lesión y el tiempo transcurrido son dos variables a considerar en el perfil de las condiciones necesarias para presentar FCD, sin embargo, existen muchos otros factores, necesarios para manifestar una escasa conciencia de las limitaciones. Del mismo modo, a medida 
que transcurre el tiempo tras la lesión, parece observarse un aumento en la conciencia de las limitaciones, sin embargo en un $57.6 \%$ de los sujetos valorados, cumplido el primer año o más de la lesión, continuaban mostrando FCD. Estos datos parecen ser acordes con la hipótesis planteada inicialmente, así como con los obtenidos por otras investigaciones donde se indica que el nivel de FCD no es constante a lo largo del tiempo sugiriendo la necesidad de realizarse una valoración de seguimiento de la FCD más allá del primer año, al considerar este periodo prematuro en la recuperación de la conciencia de los déficit (Oddy, Coughlan et al. 1985; Fleming, Strong et al. 1998).

Los resultados de estudio sugieren la influencia de una gran cantidad de variables en la presencia de FCD tras un daño cerebral, no existiendo una asociación anatómica específica relacionada con tal alteración. Así mismo se describen la severidad inicial de la lesión como factor predictivo de manifestar FCD y el tiempo tras la lesión como un factor de recuperación de dicho déficit tras la lesión. Sin embargo son necesarios más estudios para tratar de describir el resto de variables implicadas y su influencia en la presencia de FCD.

En relación con el instrumento de valoración utilizado, sería necesario una adaptación del mismo para ser utilizado por personas con severas dificultades del lenguaje, ya que dicha alteración es frecuente tras daño cerebral, pudiendo coexistir con una falta de conciencia de los déficit y sin embargo no pudiendo ser valorada adecuadamente.

Así mismo, en la línea de Giacino y Cicerone (1998) y Langer y Padrone (1992), son necesarios más estudios para tratar de diferenciar la falta de conciencia de los déficit como consecuencia directa del daño cerebral, de otras manifestaciones, aunque similares, diferentes en su tratamiento, como son la negación o minimización de las limitaciones, alteraciones más asociadas con factores de tipo psicológico.

Finalmente en este estudio se sugiere la necesidad de incorporar a las valoraciones rutinarias tras daño cerebral adquirido, una evaluación específica de la conciencia de los déficit, así como un seguimiento de dicha alteración a lo largo del tiempo, ya que el mantenimiento de este tipo de déficit condiciona en gran medida el proceso de rehabilitación y de reincorporación a la sociedad de la persona afectada de daño cerebral sobrevenido.

\section{Referencias}

Anderson S. W. y Tranel D. (1989). Awareness of disease states following cerebral infarction, dementia, and head trauma: Standardized assessment. Clinical Neuropsychologist, 3, 327-339.

Berti A., Ladavas E. y Della Corte M. (1996). Anosognosia for hemiplegia, neglect dyslexia, and drawing neglect: Clinical findings and theoretical considerations. Journal of the International Neuropsychological Society, 2, 426-40.

Bisiach E. y Geminini G. Anosognosia related to hemiplegia and hemianopia. (1991). En: G. P. Prigatano y D. L. Schacter, (Eds.). Awareness of deficit after brain injury (17-39). New York: Oxford University Press.

Borgaro S. R. y Prigatano G. P. (2003). Modification of the Patient Competency Rating Scale for use on an acute neurorehabilitation unit: The PCRSNR. Brain Injury, 17(10), 847-53.

Brickner's, R. M.(1936). The intellectual Functions of the frontal lobes. New York: Macmillan.

Chittum W. R., Johnson K., Chittum J. M., Guercio J. M. y McMorrow M. J. (1996). Road to awareness: An individualized training package for increasing knowledge and comprehension of personal deficits in persons with acquired brain injury. Brain Injury, 10, 763-76.

Deaton A. V. (1986). Denial in the aftermath of traumatic head injury: Its manifestations, measurement and treatment. Rehabilitation Psychology, $31,231-40$.

Ellis S. y Small M. (1993). Denial of illness in stroke. Stroke, 24, 757-759.

Fernández V. L. y Perea Bartolomé M. V. (2001). Evaluación neuropsicológica en el síndrome amnésico postraumático. Revista de Neurología, 16, 32 (7), 660-664.

Fleming F. y Strong J. (1995). Self-Awareness of deficits following acquired brain injury: Considera- 
tions for rehabilitation. British Journal of Occupational Therapy, 58, 55-60.

Fleming J. M., Strong J. y Ashton R. (1996). Self-awareness of deficits in adults with traumatic brain injury: How best to measure?. Brain Injury, 10(1), 1-15.

Fleming J. M., Strong J. y Ashton R. (1998). Cluster analysis of self-awareness levels in adults with traumatic brain injury and relationship to outcome. Journal of Head Trauma Rehabilitation.13(5):39-51.

García M., Sánchez I., Urrutikoetxea I., Muñoz Céspedes J. M. y Quemada J. I. (2003). Evaluación de la conciencia de déficit después de un daño cerebral: Una perspectiva neuropsicológica. Conferencia virtual presentada en el II International Congress of Neuropsychology in the Internet. Recuperado el 12 de Mayo de 2003, de http://www.serviciodc.com/congreso/congress/pass /conferences/Garcia-Valcarce.html

García M., Urrutikoetxea I., Muñoz Céspedes J. M. y Quemada J. I. (1999). Evaluación y rehabilitación neuropsicológica de la conciencia de secuelas en pacientes con daño cerebral adquirido. Conferencia virtual presentada en el First Congress on Neuropsychology in Internet. Recuperado el 20 de Febrero de2002, de http://www.serviciodc.com/congreso/congress/pass/conferences/ Garcia-Valcarce.html

German, W. J. y Fox's J. C. (1932). Observations following unilateral lobectomies. Res. Publ. Assoc. Res. Nerv. Ment. Dis, 13,378- 434.

Giacino J.T. y Cicerone K. D. (1998). Varieties of deficit unawareness after brain injury. Journal of Head Trauma Rehabilitation, 13, 1-15.

Goldberg E. y Barr W. B. (1991). Three possible mechanisms of unawareness of deficit. En G. P. Prigatano y D. Schacter (Eds.). Awareness of deficit after brain injury. (152-76). New York: Oxford University Press.

Hall K. M., Bushnik T., Lakisic Kazazic B., Wright J. y Cantagallo A. (2001). Assessing traumatic brain injury outcome measures for long-term followup of community based individuals. Archives of Physical Medicine Rehabilitation, 82 (3), 367-74.

Hartman Maeir A., Soroker N., Oman S. D. y Katz N. (2001). Anosognosia for hemiplegia in stroke re- habilitation. Neurorehabilitation and neural Repair, 15, 213- 222.

Hartman Maeir A., Soroker N., Oman S. D. y Katz N. (2003). Awareness of disabilities in stroke rehabilitation a clinical trial. Disability and Rehabilitation, 7, 25 (1), 35-44.

Hartman Maeir A., Soroker N., Ring H y Katz N. J (2002). Awareness of deficits in stroke rehabilitation. Rehabilitation Medicine, 34 (4), 158-64.

Heilbronner, R. L., Millsaps C., Azrin, R. y Mittenberg, W. (1993). Psychometric properties of the Patient Competency Rating Scale (PCRS) [ Resumen ]. Journal of Clinical and Experimental Neuropsychology, 15, 67- 68.

Heilman K. M, Barrett A. M. y Adair J. C. (1998). Possible mechanisms of anosognosia: A defect in self-awareness. Philosophical Transactions of the Royal Society of London B Biological Sciences 353, 1903-1909.

Jehkonen M, Ahonen JP, Dastidar P, Laippala P y Vilkki J. (2000). Unawareness of deficits after right hemisphere stroke: double-dissociations of anosognosias. Acta Neurológica Scandinávica, 102 (6), 378-84.

Jehkonen M., Ahonen J. P., Dastidar P., Koivisto A..M., Laippala P., Vilkki J., y Molnár G. (2001). Predictors of discharge to home during the first year after right hemisphere stroke. Acta Neurologica Scandinavica, 104, 136-141.

Johnson M. K. (1991). Reality monitoring: evidence from confabulation in organic brain disease patients. En: G. P. Prigatano y D.L. Schacter (Eds.) Awareness of deficit after brain injury. Clinical and theoretical issues (176- 97). Nueva York: Oxford University Press.

Kinsella G., Moran C., Ford B. y Ponsford J. (1988). Emotional disorder and its assessment within the severe head injured population. Psychological Medicine, 18, 57- 63.

Leathem J.M., Murphy L.J. y Flett R. A.(1998). Selfand informant-ratings on the patient competency rating scale in patients with traumatic brain injury. Journal of Clinical Experimental Neuropsychology, 20(5), 694-705.

Luria A. R. y Homskaya E. D. (1964). Disturbance in the regulative role of speech with frontal lobe le- 
sions. En J. M Warren. y A. Akert (Eds.). The frontal Granular Cortex and behavior (353- 371). New York: Mc Graw- Hill,

Luria, A. R. (1969). Frontal lobe síndromes. En P. J. Vinken y G.W. Bruyn (Eds.). Handbook of clinical Neurology (725- 757). Vol 2. Amsterdam: North Holland,

McGlynn S. y Schacter D. (1989). Unawareness of deficits in neuropsychological syndromes. Journal of Clinical Experimental Neuropsychology, 11, 143205.

Mesulam M. M (1985). Principles of behavioral neurology. Philadelphia: FA Davis.

Muñoz Céspedes J. M. (1999). Déficit Cognitivos después de un traumatismo craneoencefálico. En: Minusval. Una aproximación al tema de la discapacidad (470- 472). Madrid: Ministerio de trabajo $\mathrm{y}$ asuntos sociales.

Muñoz Céspedes J. M.(1998). Déficit cognitivos, conciencia de las limitaciones y empleo después de un daño cerebral traumático. En Libro de resúmenes del II Congreso Iberoamericano de Psicología, (33). Madrid. España.

Muñoz Céspedes J. M., Calvo B., Ríos M., García M., Sánchez A.(2002, Junio). Estructura factorial de la escala "Índice de Competencia del Paciente». Poster presentado en el II Congreso de la sociedad española de Neurorehabilitación, Barcelona, España.

Muñoz Céspedes J. M., Paúl N., Pelegrín C. y Tirapu J. (2001). Factores de pronostico en los traumatismos craneoencefálicos. Revista de neurología, 32 (4), 351- 364.

Muñoz Céspedes J. M., Quemada Ubis J. I., García Peña M., Álvarez Domínguez N. (2001, Mayo). Charasteristics of Impaired Awareness using the patient Competency Rating Scale (PCRS). Poster presentado en el 4th World Congress on Brain Injury. Turín. Italia.

Muñoz Céspedes J. M., Ríos M., Ruano A. y Moreno F. (1998). Déficit cognitivo e integración laboral de las personas con daño cerebral traumático. Polibea, 48, 38-43

Muñoz Céspedes J. M., Ríos M., Ruano A., Moreno F. (1999). Las alteraciones emocionales y la integración laboral de las personas con daño cerebral traumático. Polibea, 50, 10-16.
Noé E., Ferri J., Simó M. y Chirivella J. (2003). Conciencia de enfermedad tras un daño cerebral adquirido: Factores Pronósticos y eficacia de un programa de rehabilitación. Conferencia virtual presentada en el II International Congress of Neuropsychology in the Internet. Recuperado el 20 de Mayo de 2003, de http://www.serviciodc.com/congreso/congress/pass/conferences/Noe.html

Oddy M., Coughlan T., Tyerman A., Jenkins D. (1985). Social adjustment after head injury: A further follow- up seven years after injury. Journal of neurology, neurosurgery and psychiatry, 48, 564 - 568.

Prigatano G. P. (1991). Disturbance of self-awareness of deficit after traumatic brain injury. En: G. P. Prigatano y D. L. Schacter (Eds.) Awareness of deficit after brain injury (111-126). New York: Oxford University Press.

Prigatano G. P. (1996). Behavioural limations TBI patients tend to underestimate: A replication and extension to patients with lateralized cerebral dysfuncion. Clinical Neuropsychologist, 10, 191201.

Prigatano G. P. y Schacter D. L. (1991). Awareness of deficits after brain injury: clinical and theoretical issues. Nueva York: Oxford University Press.

Prigatano G. P. y Altman I. M.(1990). Impaired awareness of behavioral limitations after traumatic brain injury. Archives of Physical Medicine and Rehabilitation, 71(13), 1058-64.

Prigatano G. P., Bruna O., Mataró M., Muñoz Céspedes J. M., Fernández S. y Junqué C. (1998). Initial disturbances of consciousness and resultant impaired awareness in spanish patients with traumatic brain injury. Journal of Head Trauma Rehabilitation, 13(5), 1-15.

Prigatano G. P., Fordyce D. J., Zeiner H. K., et al (1986). Neuropsychological rehabilitation after brain injury. Baltimore, MD: The John Hopkins University.

Prigatano, G. P. (1997). Relación entre lesión del lóbulo frontal y disminución de la conciencia de déficit: Estudios en rehabilitación. En: C. Pelegrín, J. M. Muñoz Céspedes y J. I. Quemada (Eds.). Neuropsiquiatría del daño cerebral traumático. Barcelona: Prous Science.

Prigatano, G. P., Altaman I.M. y O’Brien, K. P. (1991). Behavioural limitations traumatic brain injury 
patients tend to underestimate. BNI Quarterly, 7, 27- 33.

Ruiz- Vargas J. M. (2000). Sobre las relaciones entre memoria y conciencia: Un enfoque neuropsicológico. Archivos de Psiquiatría, 63, 351-68.

Sachs, E. (1927). Symptomatology of a group of frontal lobe lesions. Brain, 50, 474- 479.

Schacter D. L. (1990). Toward a cognitive neuropsychology of awareness: Implicit knowledge and anosognosia. Journal of Clinical and Experimental Neuropsychology, 12, 155-78.

Sherer M., Hart T. y Nick T. G. (2003). Measurement of impaired self-awareness after traumatic brain injury: A comparison of the patient competency rating scale and the awareness questionnaire. Brain Injury, 17(1), 25-37.

Starkstein S., Fedoroff P., Price T., Leiguarda R. y Robinson R. (1993). Neuropsychological deficits in patients with anosognosia. Neuropsychiatry Neuropsychology and Behavioral Neurology, 6, 4348.

Stuss D. T. (1991).Disturbance of self-awareness after frontal system damage. En G. P. Prigatano y D. L. Schacter (Eds.). Awareness of deficit after brain injury. Clinical and theoretical issues (63-83). Nueva York: Oxford University Press.

Stuss D. T., Benson D. F. (1986). The frontal lobes. New York: Raven Press.
Teasdale G. y Jennett B. (1974). Assessment of coma and impaired consciousness. Lancet, 2, 81- 84.

Tirapu Ustárroz J., Muñoz Céspedes J. M. y Pelegrín Valero C. (2003). Hacia una taxonomía de la conciencia. Revista de neurología, 36 (11), 10831093.

Toglia J. y Kirk U. (2000). Understanding awareness of deficits following brain injury. NeuroRehabilitation; 15: 57-70.

Visser Keizer A. C., Meyboom de Jong B., Deelman B. G., Berg I. J. y Gerritsen M.J.J. (2002). Subjetive changes in emotion, cognition and behaviour after stroke: actors affecting the perception of patients and partners. Journal of clinical and experimental neuropsichology, 24(8), 1032- 1045.

Wagner M. T., Spangenberg K. B., Bachman D. L. y O'Connell P. (1997). Unawareness of cognitive deficit in Alzheimer's disease and related dementias. Alzheimer Dis Assoc Disord, 11, 125-131.

Wallace C. A., Bogner J. (2000). Awareness of deficits: emotional implications for persons with brain injury and their significant others. Brain Injury, 14(6), 549-62.

Watanabe Y., Shiel A., Asami T., Taki K. y Tabuchi K. (2000). An evaluation of neurobehavioural problems as perceived by family members and levels of family stress 1-3 years following traumatic brain injury in Japan. Clinical Rehabilitation, 14(2), 172-7. 